\title{
A slow decrementally conducting accessory pathway ablated at an unusual location: Aorto-Mitral continuity
}

\author{
mahdi Moeini ${ }^{1}$, Zahra Emkanjoo ${ }^{1}$, Farzad Kamali², and Fathemeh Jodatfar ${ }^{3}$ \\ ${ }^{1}$ Rajaie Cardiovascular Medical and Research Center \\ ${ }^{2}$ Rajaie Cardiovascular Medical and Research Center, Iran University of Medical Sciences \\ ${ }^{3}$ Shahid Beheshti University of Medical Sciences
}

May 19, 2020

\begin{abstract}
34 years old man with frequent palpitations referred to our center. Three standard diagnostic catheters were introduced through left and right femoral veins and placed in right atrium, right ventricle and coronary sinus positions. A narrow complex tachycardia with long RP-short PR could be initiated after extarstimulation from HRA following an AV nodal jump. The earliest retrograde atrial activation was recorded from His bundle position. RV apical pacing showed decremental with earliest atrial activation in His area, although no fused V-A potential was recorded in either CS or His position. His synchronous pacing from RV apex failed to advance or reset the arrhythmia. Morady maneuver by RV overdrive pacing repeatedly terminated the tachycardia. Mapping was initiated from His region and extended to all anticipated areas from parahisian region to tricuspid annulus, posteroseptal TV ring and CS. The construction of RA activation map failed to reveal earliest activation site. Mapping catheter was introduced through femoral artery and advanced to the left ventricle. At anteroseptal mitral annulus corresponding to Aorto-Mitral continuity, we recorded the earliest retrograde atrial activation (A-distal CS =50 ms). RF energy (30 W) was delivered using an irrigate tip catheter during tachycardia and resulted in termination of tachycardia immediately.
\end{abstract}

\section{Authors:}

\section{Mahdi Moeini, $\mathrm{MD}^{1,2}$}

1. Department of Cardiac Electrophysiology, Rajaie Cardiovascular Medical and Research Center, Iran University of Medical Sciences

2. Cardiac Electrophysiology Research Center, Rajaie Cardiovascular Medical and Research Center, Iran University of Medical Sciences

\section{Zahra Emkanjoo, $\mathrm{MD}^{1,2}$}

1. Department of Cardiac Electrophysiology, Rajaie Cardiovascular Medical and Research Center, Iran University of Medical Sciences

2. Cardiac Electrophysiology Research Center, Rajaie Cardiovascular Medical and Research Center, Iran University of Medical Sciences

\section{Farzad Kamali, $\mathrm{MD}^{1,2}$}

1. Department of Cardiac Electrophysiology, Rajaie Cardiovascular Medical and Research Center, Iran University of Medical Sciences

2. Cardiac Electrophysiology Research Center, Rajaie Cardiovascular Medical and Research Center, Iran University of Medical Sciences 


\section{Fathemeh Jodatfar, MD ${ }^{1}$}

Cardiovascular Research Center, Taleghani Hospital, Shahid Beheshti University of Medical Sciences

\section{Correspondence and reprint request:}

Mahdi Moeini, Department of Cardiac electrophysiology, Rajaie Cardiovascular Medical and Research Center, Vali-Asr St., Ayatollah Hashemi-Rafsanjani Blvd, Tehran, 1996911151 Iran

Tel: +982123923822

Fax: +98212392 2340

Abstract

A 34 years old man with refractory symptoms and unresponsive to antiarrhythmic drug therapy referred to our center. Electrophysiological study and diagnostic maneuvers confirmed the diagnosis of orthodromic reciprocating atrioventricular tachycardia using a slowly and decrementally conducting AP as orthodromic arm and slow AV nodal pathway as antidromic arm. AP was successfully ablated radiofrequency application through retrograde approach at Aorto- mitral continuity. The response of this arrhythmia to diagnostic maneuvers at an unusual location of AP offers insight to electrophysiological properties of this type of accessory pathways.

Introduction

Slowly and decrementally conducting accessory pathways (Aps) responsible for induction of a specific type of reciprocating reentrant tachycardia called permanent junctional reciprocating tachycardia (PJRT).(1) PJRT often in the form of incessant tachycardia more commonly seen in early ages. The tachycardia is characterized by a narrow QRS rhythm with variable rates, a retrograde $\mathrm{P}$ wave axis, and an RP interval that is longer than the PR interval. In many patients, PJRT is fairly difficult to control medically. Catheter ablation has now become as a highly effective technique for eliminating these accessory pathways with minimal risk of AV block (2). Although the anatomic origin in majority of cases are localized to posteroseptal area but few cases with uncommon location has been reported. In this report we introduce an uncommon location with electrophysiological properties at Aorto-Mitral continuity.

Case presentation

A 34 years old man with history of frequent palpitations refractory to drug therapy referred to our center. He had documented tracing of a narrow QRS tachycardia terminated after Adenosine injection. Clinical examination, chest $\mathrm{x}$-Ray and echocardiography revealed no abnormality.

Patient brought to electrophysiology Lab after obtaining formal consent, in sinus rhythm. Procedure was performed under conscious sedation. Antiarrhythmic drugs were withdrawn five days before procedure.

Three standard quadripolar and decapolar diagnostic catheters were introduced through left and right femoral veins and placed in right atrium (HRA), right ventricle (RV) and coronary sinus (CS) positions. His bundle (HB) potential was recorded using mapping catheter. Twelve lead ECG during sinus rhythm showed normal PR interval with no manifest pre-excitation. The basic parameters of AH and HV interval were 85msec and $49 \mathrm{msec}$, respectively. There was the evidence of dual AV node physiology manifested as atrioventricular (AV) nodal jump. A narrow complex tachycardia with long RP-short PR could be initiated after extarstimulation from HRA following an AV nodal jump. The earliest retrograde atrial activation during tachycardia was recorded from His bundle position. RV apical pacing showed decremental with earliest atrial activation at the His area, although no fused V-A potential was recorded in either CS or His position. Notably, we were able to induce the arrhythmia with RV apical extrastimulation.

Diagnostic maneuvers were performed. His synchronous pacing from RV apex failed to advance or reset the arrhythmia on multiple attempts. However, Morady maneuver by RV overdrive pacing repeatedly terminated the tachycardia. 
a avi

v1

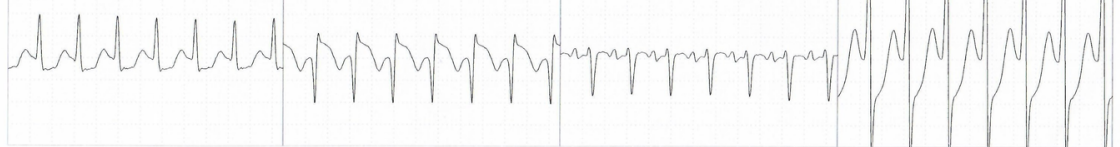

II

"

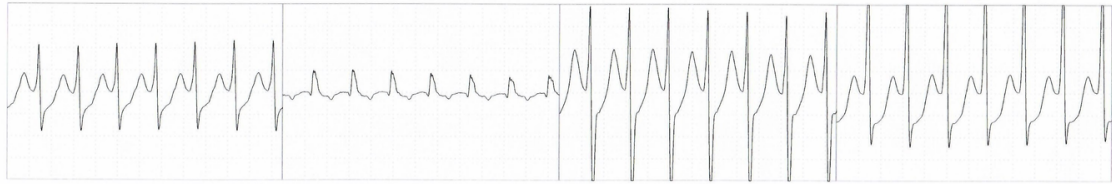

av

ram

III

my
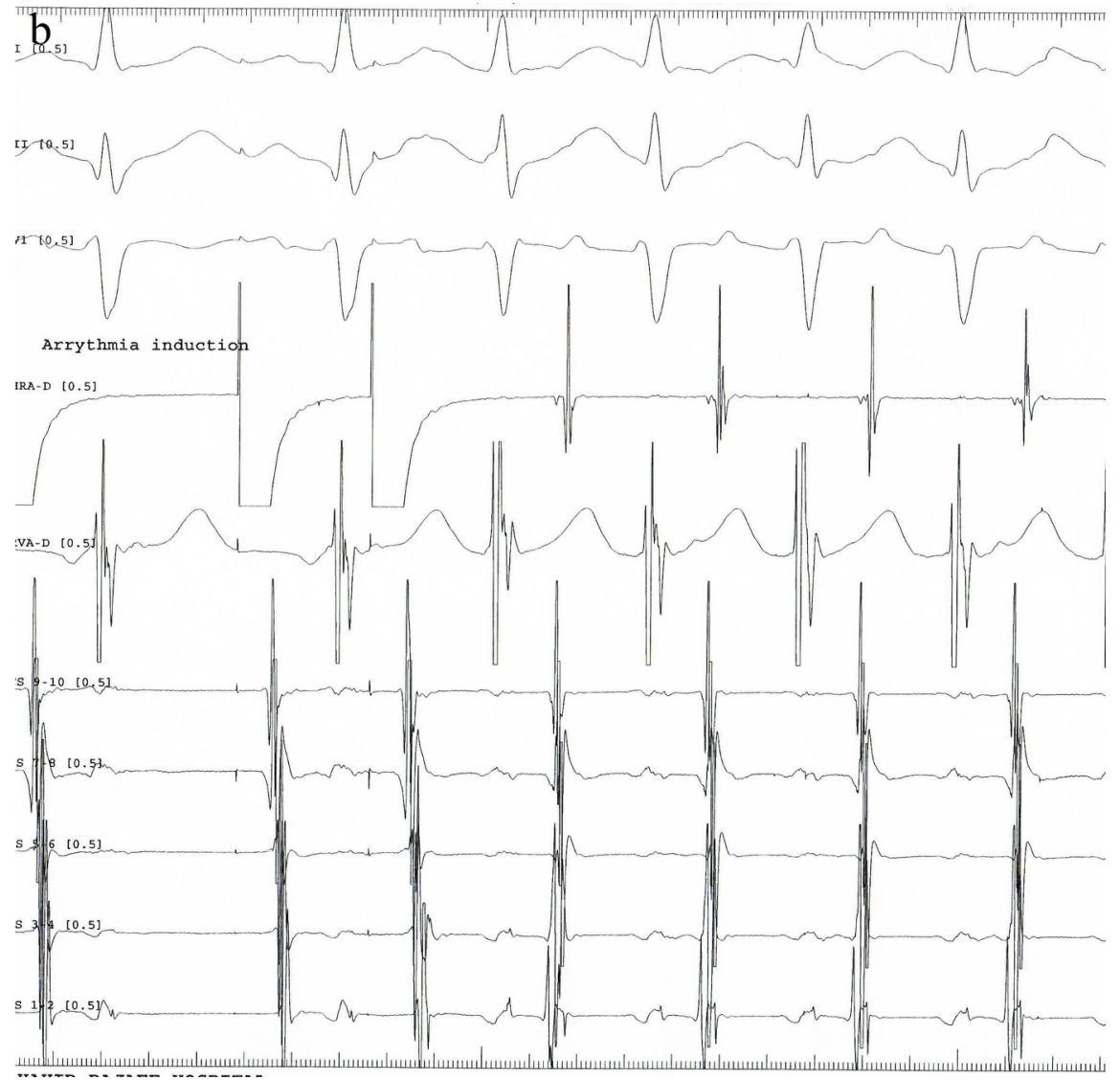


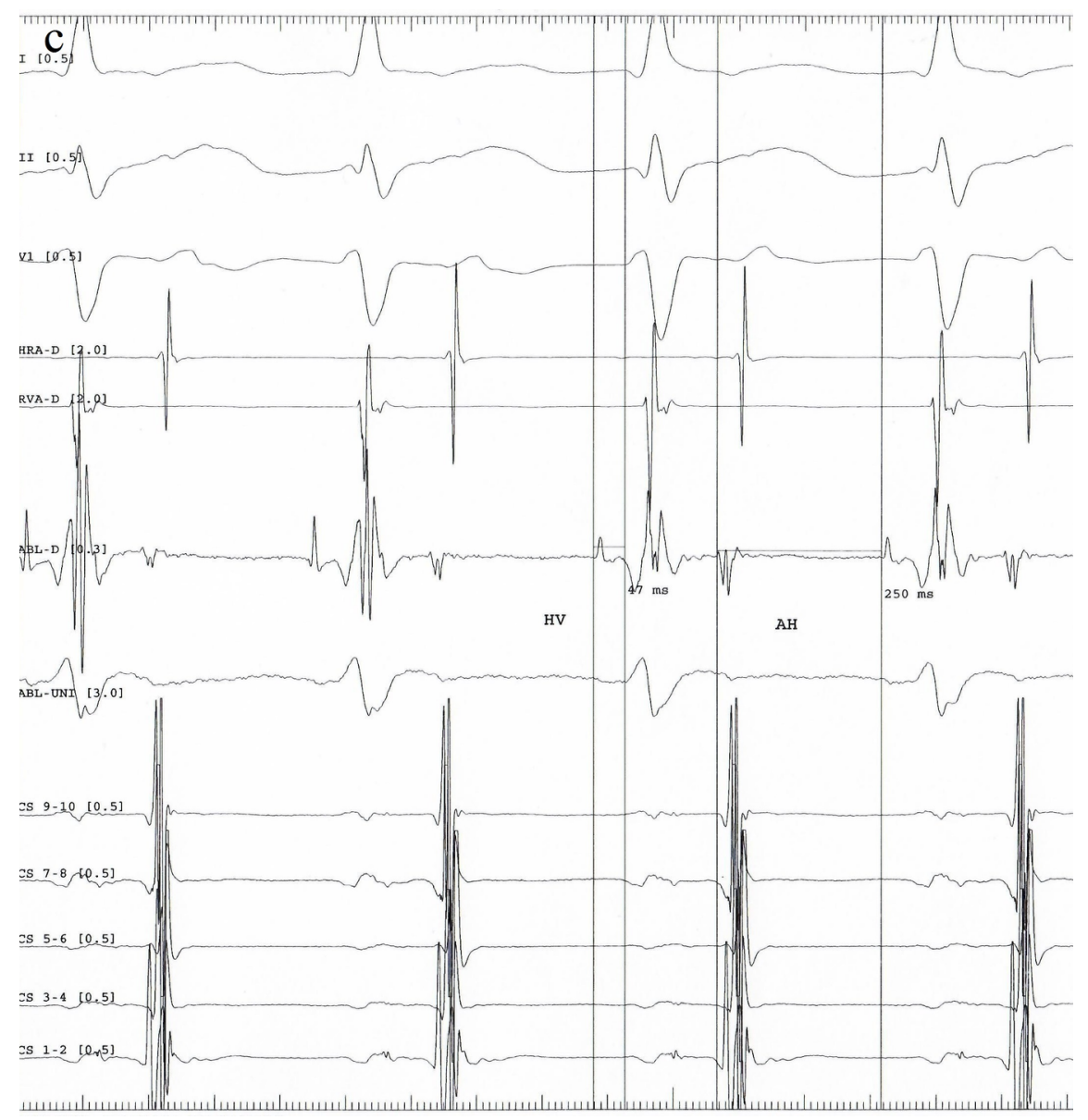




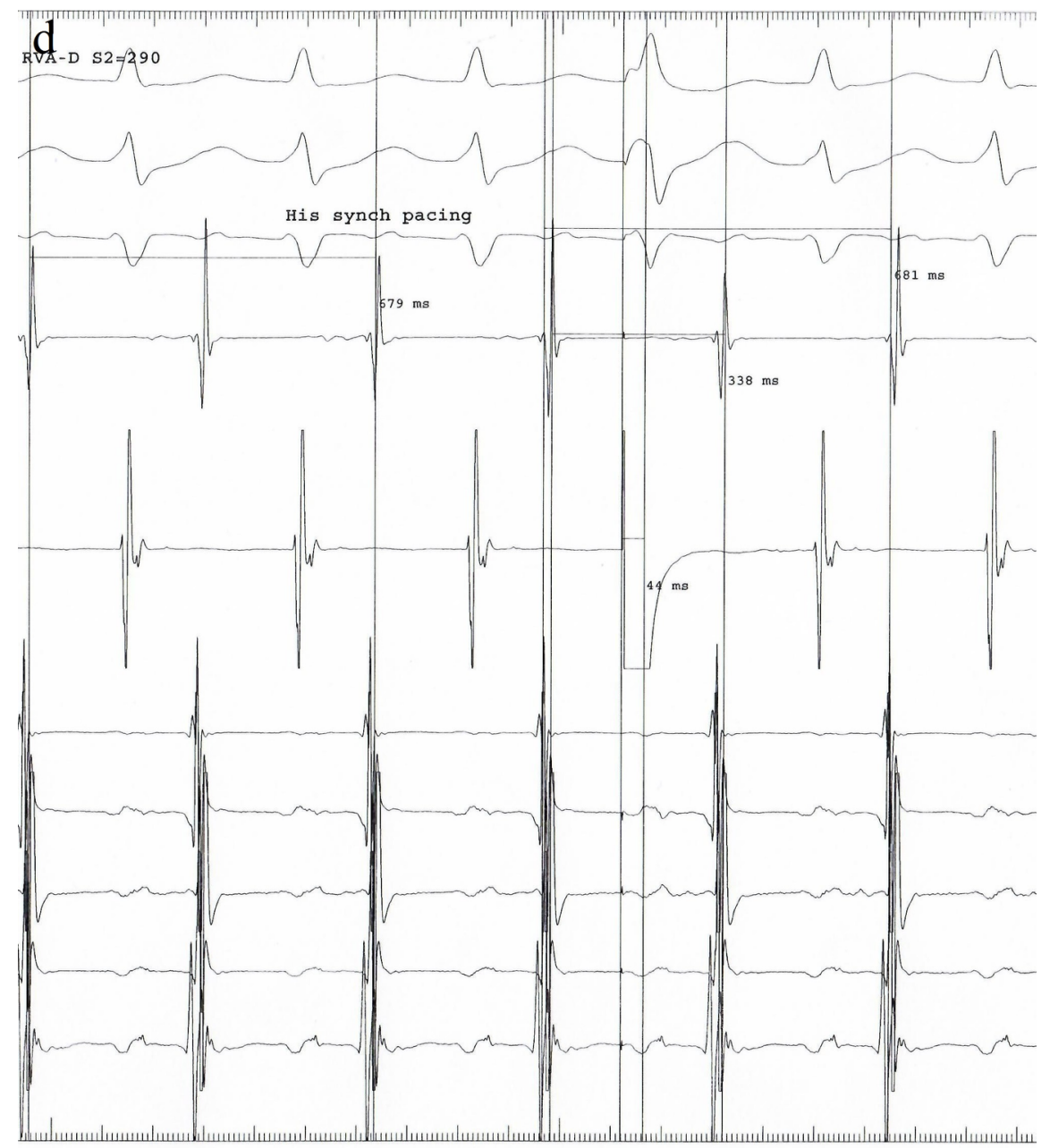

Figure1: Surface ECG of narrow complex tachycardia (a) arrhythmia initiation after premature stimulation from HRA with AV prolongation (b) AH and HV interval during tachycardia 47 and 250 msec respectively(c) His synchronous pacing with fusion beat that failed to advance the arrhythmia (d)

Based on observation during electrophysiologic study (EPS), alteration in V-V interval preceded A-A changes with fixed V-A interval. This was against atrial tachycardia.

Due to inability to reset the arrhythmia and considering the existence of decremental properties and arrhythmia induction following AV nodal jump and AH prolongation, the existence of an AP couldn't be proved.

We proceeded by mapping the earliest retrograde atrial activation using a 4-mm non-irrigated tip ablation catheter. Mapping was initiated from His region and extended to all anticipated areas from parahisian region to tricuspid annulus, posteroseptal TV ring, CS ostium and proximal CS at the roof and base of CS and in middle cardiac vein. The construction of RA activation map failed to reveal earliest activation site. So, we decided to map the left side. Mapping catheter was introduced through femoral artery and advanced retrogradely to the left ventricle (LV). Mapping was started from Aortic cusps then the catheter was pushed down inside LV cavity beneath the Aortic cusps, at the anteroseptal mitral annulus corresponding to Aorto-Mitral continuity (AMC), we recorded the most earliest retrograde atrial activation relative to the aforementioned locations (A-distal $\mathrm{CS}=50 \mathrm{~ms}$ ). 
Although the above position had the most fused A-V potential, but a distinct isoelectric line could be recorded between A-V potentials. RF energy $(30 \mathrm{~W})$ was delivered using an irrigate tip catheter at this site during tachycardia and resulted in termination of tachycardia after few seconds.
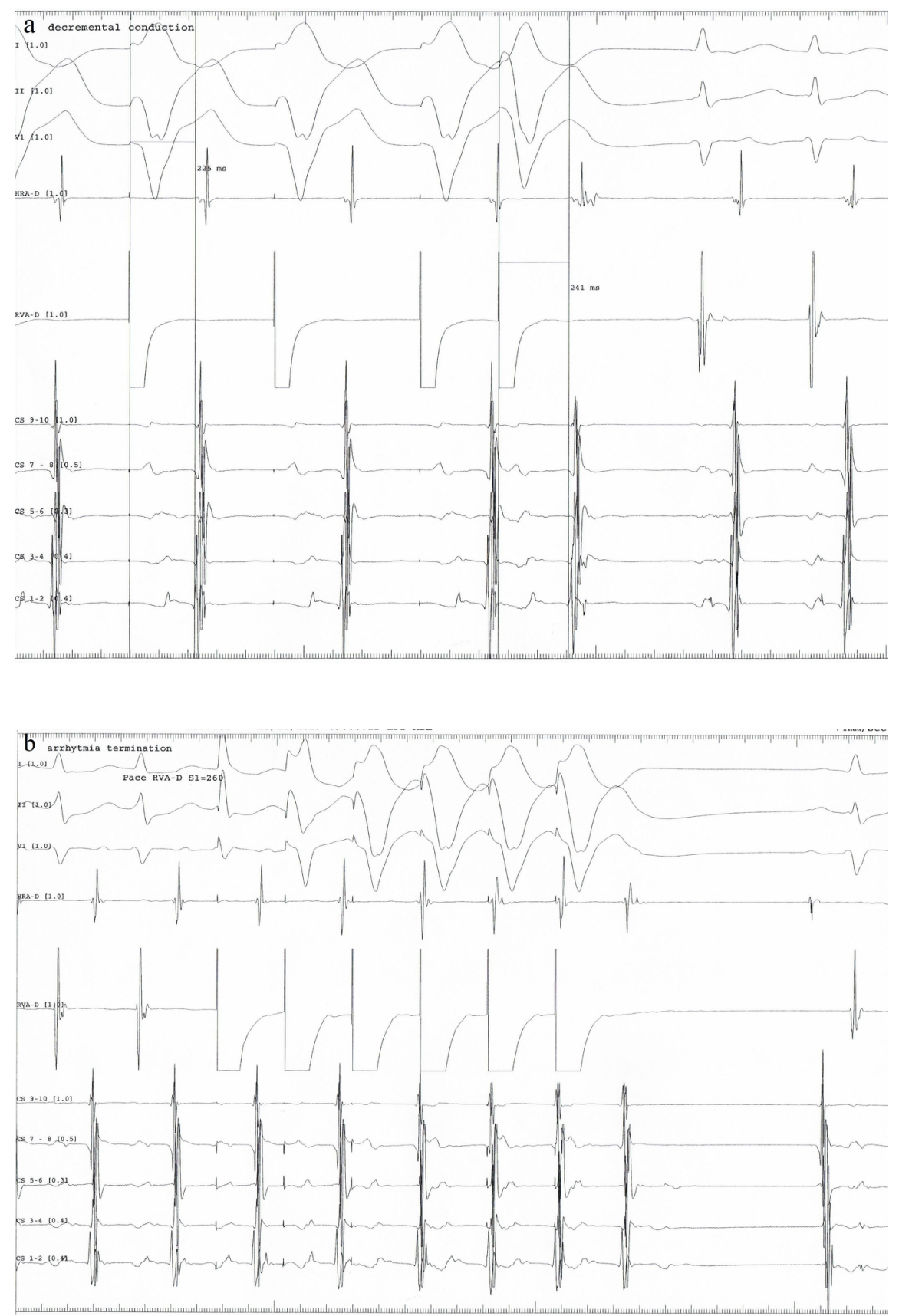

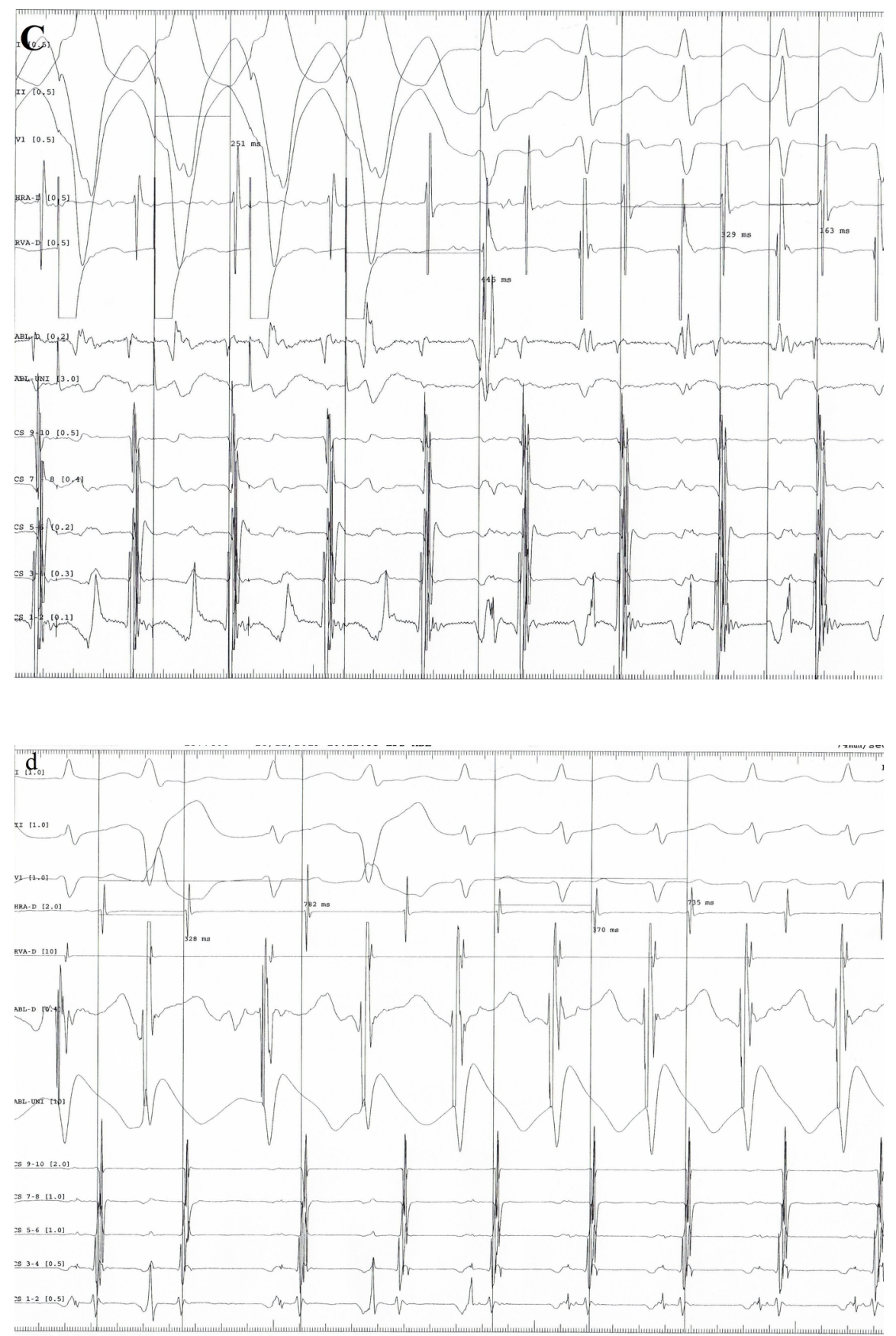

Figure2: Decremental conduction through AP, VA interval increased from 225 to 241 msec with no change in CS sequence after RVA extrastimulation (a) Arrhythmia termination at the first fusion beat with no alteration in CS activation and perpetuation of decremental retrograde conduction through AP (b)Morady Maneuver with PPI-TCL(445-329)>115 and SA-VA(251-163)>85 consistent with AVNRT (c) Advancement and resetting after catheter induced PVC during AMC mapping(d)

Thereafter, there was V-A dissociation during RV apical pacing. 

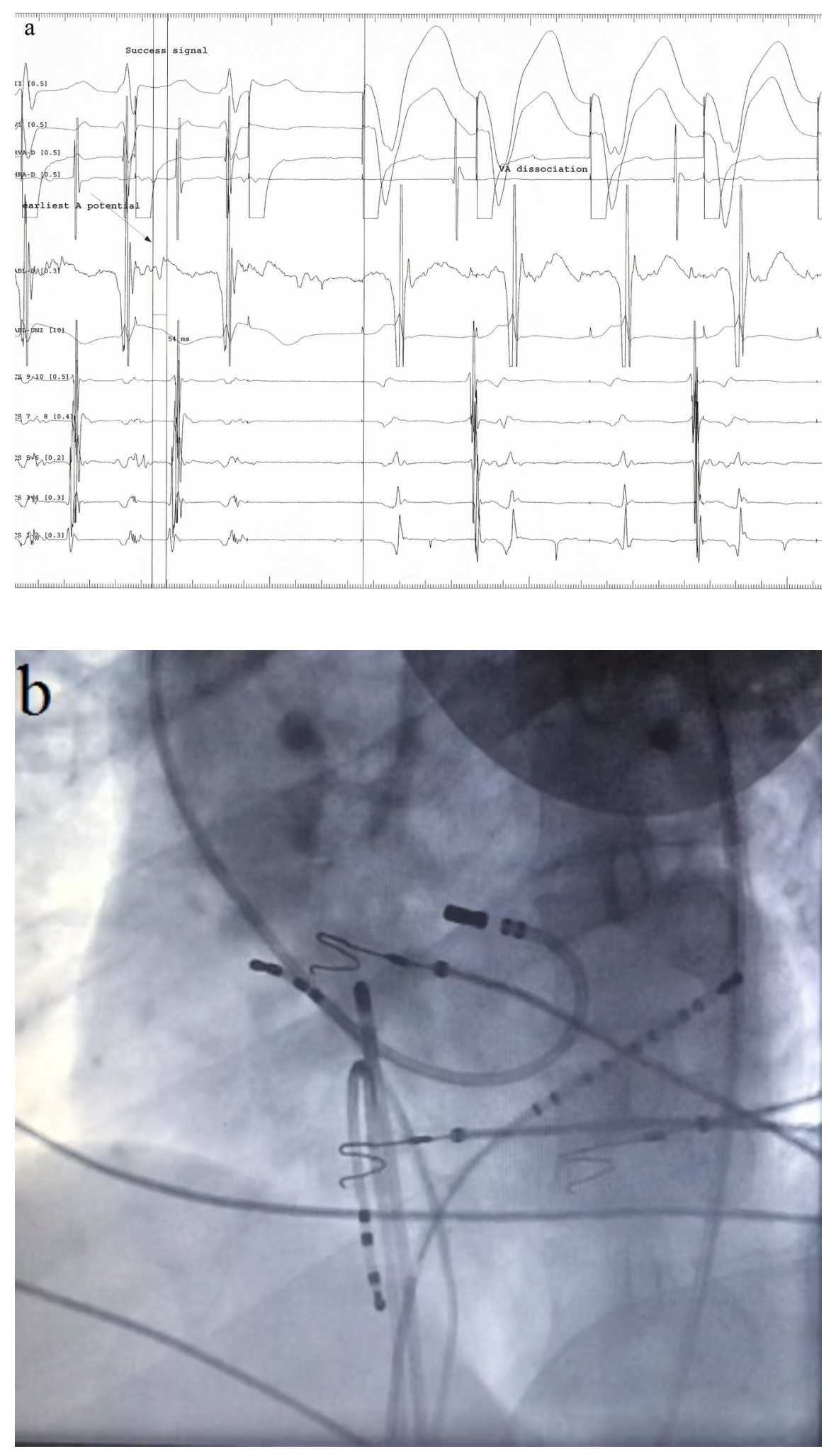


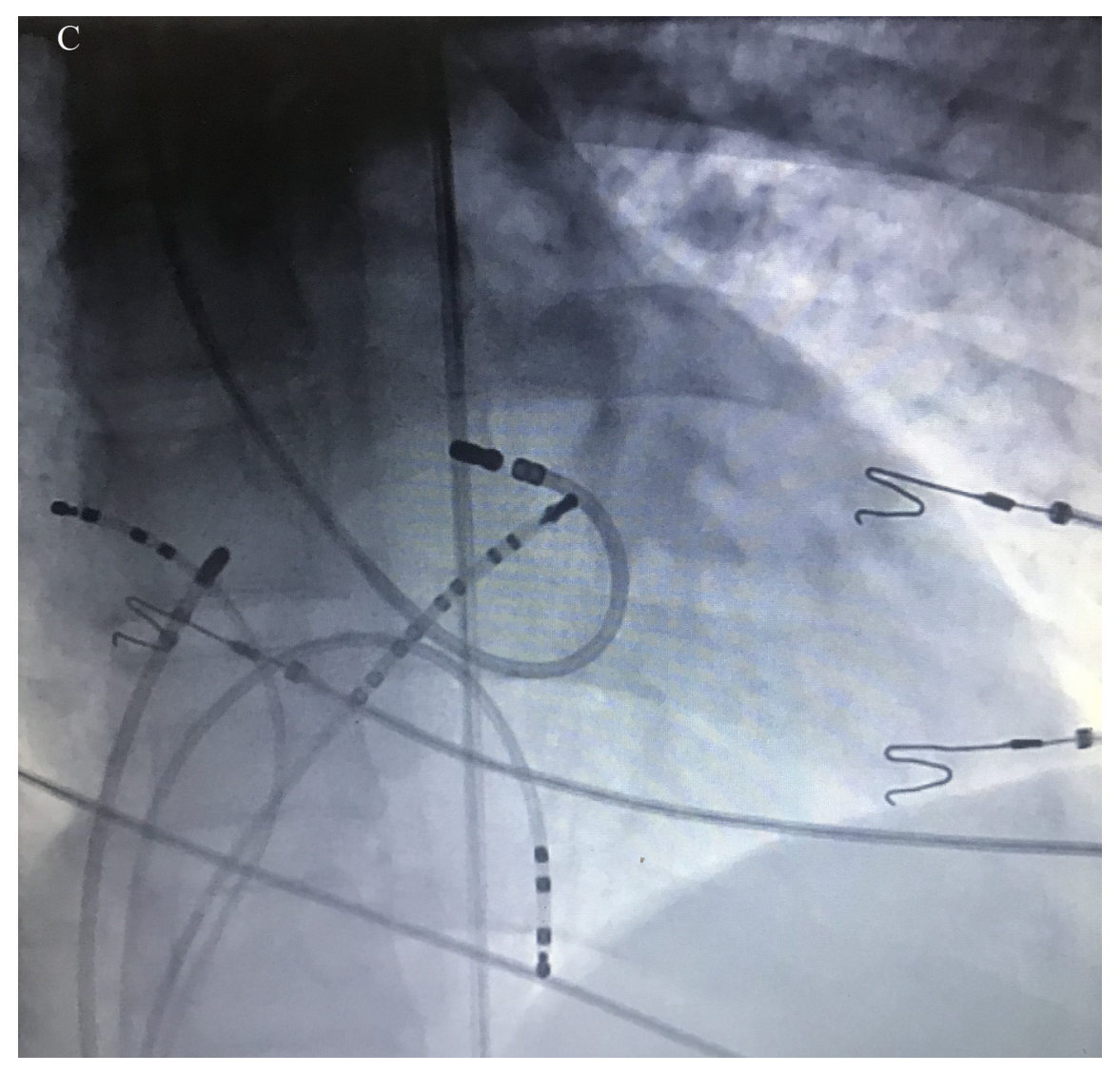

Figure3: The earliest retrograde atrial activation at AMC with 50msec earlier relative to distal CS terminated the arrhythmia and resulted in VA dissociation (a) The fluoroscopic view of successful site of ablation at LAO and AP projection at Aorto-mitral continuty

During post ablation study, 30 minutes later, there was no evidence of AP conduction. AH and HV intervals were 80 and $50 \mathrm{msec}$, respectively. The patient was free of palpitation during 3 months post ablation follow up.

\section{Discussion}

PJRT is in differential diagnosis with other two types of arrhythmia with long RP-short PR interval including atypical atrioventricular nodal tachycardia (AVNRT) and Atrial tachycardia. In this case, we easily ruled out atrial tachycardia by observing arrhythmia termination with ventricular overdrive pacing and V-V interval changes preceding A-A interval during tachycardia. His synchronous pacing could not reset nor advance the arrhythmia. Decremental conduction during ventricular and HRA extrastimulation and AV nodal jump during HRA extrastimulation was recorded during study. Entrainment response from ventricular site with indexes of (PPI-TCL) and (SA-VA) were consistent with AVNRT $(3,4)$. However, Alteration in CS activation sequence during arrhythmia initiation could challenge the diagnosis of AVNRT.

Not surprisingly, the mapping catheter induced PVC during catheter manipulation in LVOT did reset the arrhythmia (Fig 2d), in close proximity to the earliest site of retrograde atrial activation and successful ablation site at Aorto- Mitral continuity.

The findings during EPS were consistent with decremenatal properties of AP. In the presence of decremental properties of AP, it is often impossible to confirm an AP as the retrograde limb using the standard technique 
of atrial preexcitation by a ventricular premature beat while the His is refractory, because the retrograde conduction decrements after premature ventricular stimulation (5).

This AMC separates the LV myocardium and the LVOT from the LV inflow. The AMC also relates to the LA of the noncoronary sinus of valsalva.(6) Albeit that the region of the AMC consists of a membranous structure and without any significant neighboring myocardium, some data suggests that remnant fascicles of Purkinje tissue are related to the conduction system, which may be the source of abnormal signals that have been successfully targeted for ablation.(7)

Contrary to few reports of PJRT and concealed AP ablated at left anteroseptal area from transseptal approach (8-10), we used retrograde approach for mapping the region. As a result the V: A ratio at successful ablation site was 8:1 delineating the ventricular insertion site of AP. In comparison to transseptal approach that localized the atrial insertion site near His bundle catheter, ventricular insertion was far apart from His bundle. This safe position was accompanied with minimal risk of AV block and seemed to be preferred.

Another unique characteristic of this tachycardia was participation of slow pathway in the path of arrhythmia which resulted in lengthening of antidromic arm and consequently much less delay in orthodromic arm. Subsequently unlike typical PJRT with long RP-short PR, we encountered an arrhythmia that atrial potential was recorded at mid $\mathrm{V}-\mathrm{V}$ interval.

In summary, we present an unusual form of PJRT whose ventricular insertion was successfully ablated in the AMC.

References:

1. Coumel P, Cabrol C, Fabiato A, Gourgon R, Salma R. Tachycardie permanentepar rythme reciproque. Arch Mal Coeur. 1967;60:1830-1864.

2. Haïssaguerre M, Montserrat P, Warin JF, Donzeau JP, Le Metayer P, Massiere JP. Catheter ablation of left posteroseptal accessory pathways and of long RP' tachycardias with a right endocardial approach.Eur Heart J . 1991;12:845-859.

3. Knight BP, Ebinger M, Oral H, et al. Diagnostic value of tachycardia features and pacing maneuvers during paroxysmal supraventricular tachycardia. J Am Coll Cardiol . 2000;36:574-582.

4. Michaud GF, Tada H, Chough S, et al. Differentiation of atypical atrioventricular node re-entrant tachycardia from orthodromic reciprocating tachycardia using a septal accessory pathway by the response to ventricular pacing. J Am Coll Cardiol . 2001;38:1163-1167.

5. Haïssaguerre M, Montserrat P, Warin JF, Donzeau JP, Le Metayer P, Massiere JP. Catheter ablation of left posteroseptal accessory pathways and of long RP' tachycardias with a right endocardial approach.Eur Heart J . 1991;12:845-859.

6. Shehata M, Liu T, Joshi N, Chugh SS, Wang X. Atrial tachycardia originating from the left coronary cusp near the aorto-mitral junction: anatomic considerations. Heart Rhythm . 2010;7:987-991.

7. Hai JJ, Chahal AA, Friedman PA, et al. Electrophysiologic characteristics of ventricular arrhythmias arising from the aortic mitral continuity- potential role of the conduction system. $J$ Cardiovasc Electrophysiol . 2015;26:158-163.

8. Baruch S. Ticho, MD, PhD, J. Philip Saul, MD, J. Edward Hulse, MD, Wang De, MD, Janice Lulu, RN, and Edward P. Walsh, MD. Variable Location of Accessory Pathways Associated with the Permanent Form ofJunctional Reciprocating Tachycardia and Confirmation with Radiofrequency Ablation. (Am J Cardiol 1992;70:1559-1564)

9. Tada.H, Naito. S, Taniguchi. K, Nogami. A. Concealed Left Anterior Accessory Pathways: Two Approaches for Successful Ablation.J Cardiovasc Electrophysiol, February 2003, Vol. 14, pp. 204-208. 
10. Ali.H, Vitali-Serdoz.L, Ferrero.P, Pittalis.M, Belotti.G, Cappato.R.An unusual case of permanent junctional reciprocating tachycardia: Successful ablation at the mitral annulus-aorta junction(J Interv Electrophysiol 2008;23:213-217)

\section{Hosted file}

Doc1.docx available at https://authorea.com/users/323964/articles/452383-a-slowdecrementally-conducting-accessory-pathway-ablated-at-an-unusual-location-aorto-mitralcontinuity 